\title{
Operational Procedures to Obtain High Beam-beam Tune Shifts in LEP Pretzel Operation
}

\author{
R. Bailey, P. Collier, T. Bohl, H. Burkhardt, K. Cornelis, G. De Rijk, A. Faugier, \\ M. Jonker, M. Lamont, G. Roy, H. Schmickler and J. Wenninger, \\ CERN, Geneva, Switzerland
}

\begin{abstract}
During the running period 1994 the luminosity of LEP has been improved without colliding higher bunch currents. This paper describes the main procedures used by the operations group for luminosity optimization. Separator scans in the vertical plane and orbit interpolations in the horizontal plane are used to adjust settings for electrostatic separators in order to ensure headon collisions. The closed orbit is corrected towards a reference orbit (called "golden" orbit) which has been found empirically to produce good luminosity. The settings of the skew quadrupoles for coupling compensation is obtained by measuring the closest tune approach of the horizontal and vertical betatron tunes.
\end{abstract}

\section{Introduction}

In 1994 LEP was operated since the beginning of the year using a horizontal Pretzel scheme with $8 e^{+} \times 8 e^{-}$bunches. The beams are collided at 4 interaction points (IPs) where high energy particle detectors are installed. The horizontal pretzel orbits, used to separate the two beams at 8 mid-arc collision points, are produced with 8 unipolar electrostatic separators operated at a field of $1 \mathrm{MV} / \mathrm{m}$.

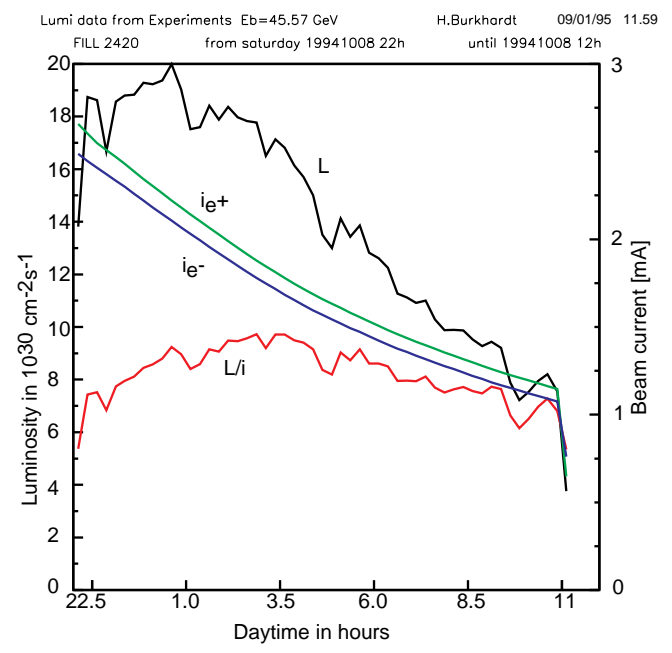

Figure. 1. Evolution of the luminosity, the beam currents and the ratio luminosity over beam current during a good LEP coast.

In 1994 the bunch currents reached typically $0.32 \mathrm{~mA}$ at the beginning of the coasts, corresponding to a total beam current of about $5.2 \mathrm{~mA}$. These values are not significantly higher than for the 1993 run [1]. Despite this fact, a total integrated luminosity of $60 p b^{-1}$ was delivered to each of the four exper- iments in 1994, 50\% higher than in 1993 although the length of the runs and the efficiencies were almost identical. This improvement was achieved mainly by increasing the average vertical beam-beam tune shift $\xi_{y}$ from 0.03 to 0.04 . Peak luminosities of $2.2 \cdot 10^{31} \mathrm{~cm}^{-2} \mathrm{~s}^{-1}$ were obtained at the beginning of fills. Figures 1 and 2 show the evolution of the luminosity and $\xi_{y}$ for a good coast. At lower beam currents the beam lifetimes are limited by beam-beam-Bremsstrahlung and are then correlated with the beam-beam tune shift [2].

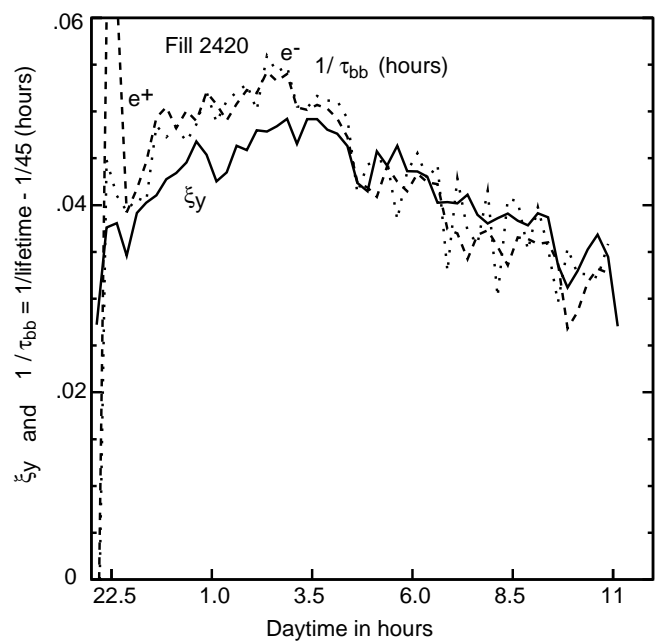

Figure. 2. Evolution of the vertical beam-beam tune shift $\xi_{y}$ in the same coast that is shown in figure 1. After a short time $\xi_{y}$ settles down above 0.04 . The beam lifetimes in collision $\tau_{b b}$ are correlated to $\xi_{y}$ because they are limited by the beam-beam Bremsstrahlung, particularly at lower beam currents. The single beam lifetime of 45 hours has been subtracted from the lifetimes shown in the figure.

\section{Separator Adjustments}

The energy sawtooth in LEP together with machine errors induces non-closures of the vertical separator bumps used to avoid collisions in 4 interaction points where no experimental detector is installed. This non-closure as well as coupling of the horizontal Pretzel orbits can lead to vertical collision offsets at the 4 IPs where the beams are put into collision.

To optimize the luminosity, adjustments of vertical trim separators are used to steer the beams against each other to minimize vertical collision offsets. Dedicated luminosity detectors provide fast luminosity measurements in the control room every $20 \mathrm{sec}$ onds. This information is used to perform scans or fine tunings of 


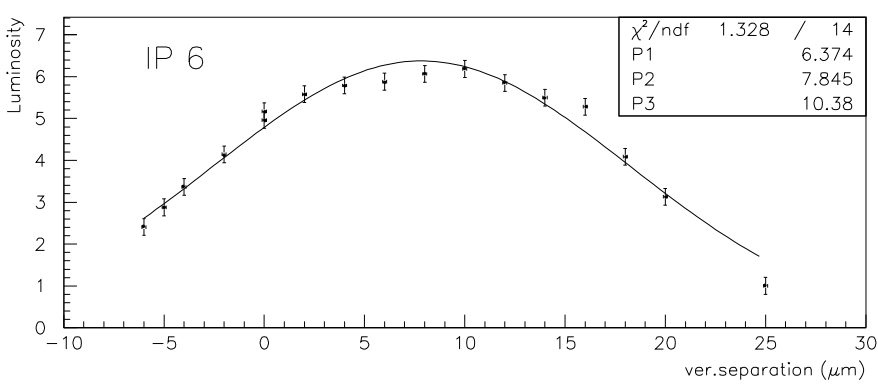

Figure. 3. A complete vertical separator scan around IP number 6. The luminosity is displayed as a function of the vertical separator trim. A Gaussian distribution has been fitted to the data points. The optimum separator settings corresponds to $7.9 \mu \mathrm{m}$. The width of the distribution is $10.4 \mu \mathrm{m}$

the separator settings. Figure 3 shows an example of a complete scan. Once the optimum position is known, some fine tuning of $1-2 \mu \mathrm{m}$ is made on a fill by fill basis, usually while the 4 experiments are already taking data. Typical corrections did not exceed $10 \mu \mathrm{m}$ at the IP.

The non-closure of the horizontal Pretzel orbits can be corrected to avoid horizontal collision offsets with two horizontal trim separators. A fit of the $e^{+} e^{-}$difference orbit is used to calculate the collision offsets and the correction trim for the separators. An example of an orbit extrapolation is shown in figure 4. This technique allows to reduce the horizontal offsets to less than one tenth of the horizontal RMS beam size at the IP.

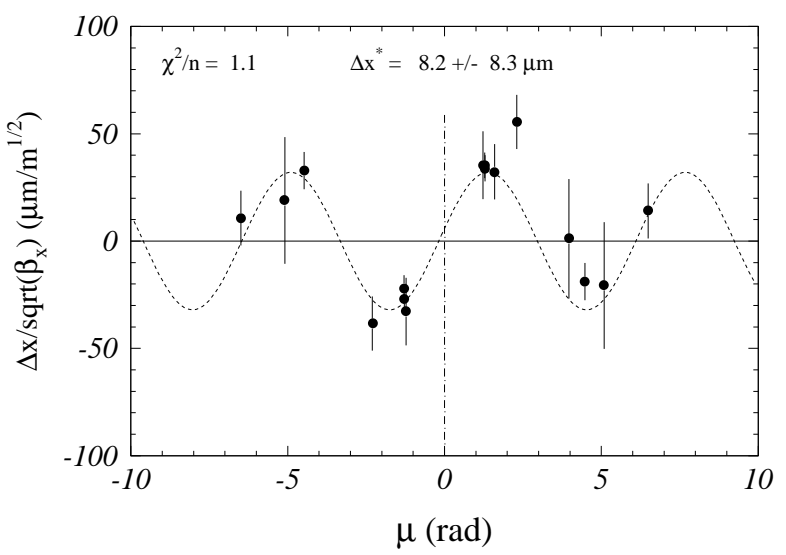

Figure. 4. Example of a fit of the horizontal $e^{+} e^{-}$difference orbit after adjustment of the horizontal trim separators. The horizontal difference orbit, normalized by the square root of the horizontal betatron function $\beta_{x}$, is shown as a function of the phase advance $\mu$ with respect to the IP. The fitted horizontal offset $\Delta \boldsymbol{x}^{*}$ at the IP is $8.2 \pm 8.3 \mu \mathrm{m}$.

\section{Golden Orbit}

The LEP quadrupoles were completely realigned for the first time during the winter shutdown 1992/1993. High levels of po- larization and a better control of the vertical orbit were achieved as a result of this operation [3]. To maintain this favorable situation all quadrupoles were again aligned vertically in the shutdown 1993/1994 to correct for the continuous position drifts due to ground motion [4].

During the first months of the 1994 LEP run a "golden" orbit yielding very high beam-beam tune shifts of about 0.04 was found empirically. The most common method to find such an orbit was to use 'bare orbit' correction strategies. With this method the theoretical orbit is computed from a measured orbit by reseting to zero all correctors. A MICADO [5] type correction on the result is then used to establish a new set of correctors. It was not sufficient to reach the smallest vertical orbit RMS to obtain the best luminosity performance. The vertical(horizontal) RMS at the pickups of the average $e^{+} e^{-}$beam position for the "golden" orbit is about $0.45(0.5) \mathrm{mm}$.

In addition to the pickup readings, the corrector settings were found to play a significant role. The corrector settings of both planes were therefore saved at the beginning of each fill to be reloaded at the start of the following fill. The orbit resulting from the reloading was then corrected towards the "golden" orbit. This procedure gave reproducible performances for the whole 1994 run. The importance of the corrector settings is related to the closed orbit correction strategy (bare, number of correctors...). A study performed during the 1993 run [6] demonstrated that a smaller residual machine coupling can be achieved with a "golden" orbit. The same study showed that the average vertical dispersion in the whole LEP ring is not a crucial parameter for a good orbit as long as it is below $10 \mathrm{~cm}$. But in order to achieve the highest possible beam-beam tune shifts the local vertical dispersion at the IPs plays a certain role [6]. This is confirmed by the fact that local dispersion bumps have been successfully used to tune the luminosity.

The vertical orbit at LEP exhibits large and sometimes fast drifts. After periods of 12 hours the RMS drift can reach a few $\mathrm{mm}$. It could be shown that these drifts are caused by vertical motion of the super-conducting low-beta quadrupoles installed next to the experimental interaction points [7], [8]. The instability of their vertical position (a few tens of $\mu \mathrm{m}$ ) is related to their support girder which is attached to the ground on one end only because the quadrupoles are embedded in the experimental detectors. To maintain high beam-beam tune shifts, frequent orbit corrections have to be performed. In 1994 a total of about 13200 vertical orbit corrections were made during the running period [7].

A dynamic beam-based alignment technique [9] has been used in 1994 to measure systematic position offsets between the center of the quadrupoles and of the pickups in the regions close to the interaction points. These measurements unveiled large systematic offsets of up to $2 \mathrm{~mm}$. Once the measured beam positions had been corrected for these offsets, it was found that the "golden" orbit had in fact been empirically centered in the quadrupole around the interaction points.

\section{Coupling Compensation}

The coupling is measured at LEP using the closest tune approach of the betatron tunes [11]. The two betatron tunes are crossed while the Q-meter is measuring continuously in PLL 


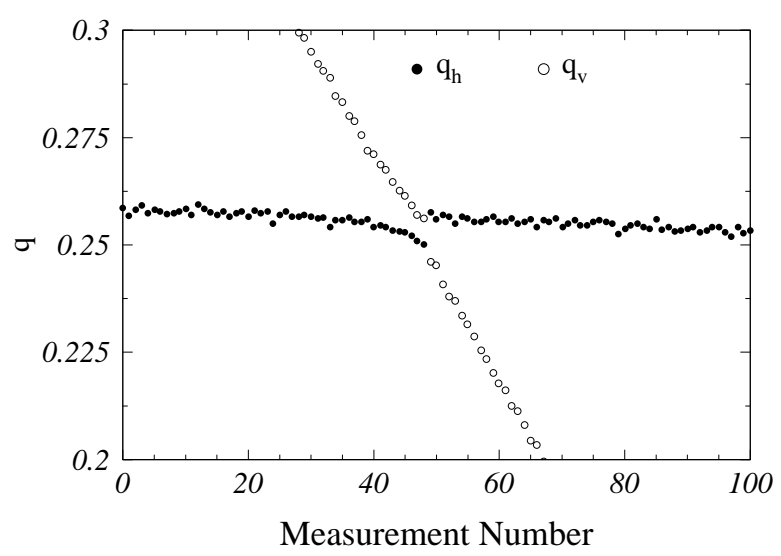

Figure. 5. Evolution of the horizontal and vertical tunes during a closest tune approach measurement. The closest approach can be clearly seen on this figure.

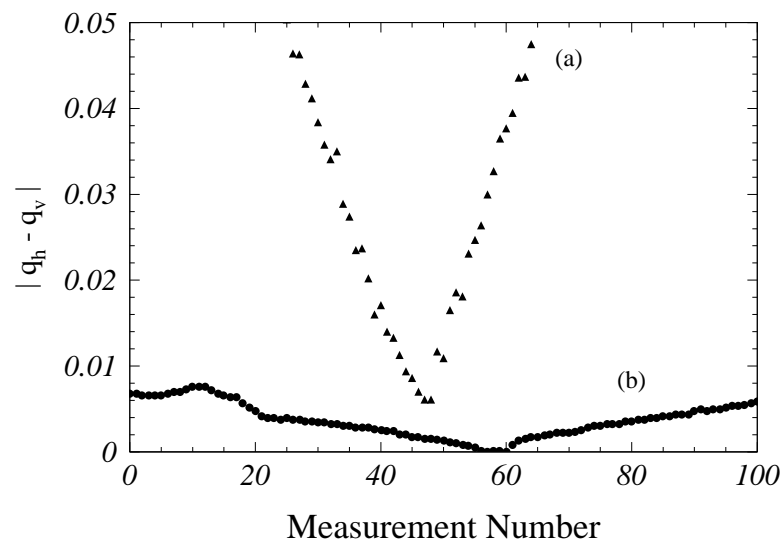

Figure. 6. Difference between the horizontal and vertical tunes for two closest tune approach measurements. Curve (a), where the coupling compensation is poor, corresponds to the measurement shown in figure 5. The lower curve (b) shows a measurement for a machine with low residual coupling between the two planes.

mode as shown in figure 5 . The smallest distance $\Delta q$ between the tunes measures the strength of the coupling resonance. Figure 6 shows the difference between the two betatron tunes for the measurements of a poorly (a) and a very well compensated machine (b). The compensation of the coupling is adjusted with the skew quadrupoles to reach the smallest possible distance between the two betatron tunes during the closest tune approach measurements. As has already been pointed out in the previous section, a very small residual coupling can be achieved with a "golden" orbit [6]. In the absence of vertical dispersion the emittance ratio $\kappa\left(\kappa=\varepsilon_{v} / \varepsilon_{h}\right)$ is related to the closed tune approach by :

$$
\kappa=1-\frac{2 r^{2}+1}{4 r^{2}+1}
$$

where

$$
r=\frac{\Delta q}{2\left|q_{h}-q_{v}\right|}
$$

The distance to the main coupling resonance is $\left|q_{h}-q_{v}\right|=0.14$ for LEP which is operated in physics with tunes $q_{h}=90.3$ and $q_{v}=76.16$. To obtain a high vertical beam-beam tune shift, the emittance ratio must be kept below the betatron function ratio at the IP of $2.5 \%\left(\beta_{h}^{*}=2 \mathrm{~m}, \beta_{v}^{*}=5 \mathrm{~cm}\right)$. This limit is achieved in all cases of vertical orbit correction.

\section{Conclusion}

The 1994 LEP run has been very successful since it saw a $50 \%$ increase of the delivered integrated luminosity over the 1993 run. This was possible with the help of numerous optimization procedures. Collision offsets have been corrected with trim separators using the online luminosity and closed orbit information. Small vertical beam sizes very obtained by a careful minimization of the residual machine coupling. A reproducible performance was achieved by using "golden" orbits. Vertical beambeam tune shifts larger than 0.04 have been achieved in daily operation.

\section{References}

[1] R. Bailey et al., "LEP Operation in 1993 with the Pretzel Scheme", Proc. EPAC, London, 1994.

[2] H. Burkhardt and R. Kleiss, "Beam Lifetimes in LEP", Proc. EPAC, London, 1994.

[3] R. Assmann et al., "Recent Achievements in Transverse Spin-Polarisation at LEP", Proc. EPAC, London, 1994.

[4] M. Hublin et al., "Realignment of LEP in 1993-1994", Proc. EPAC, London, 1994.

[5] B. Autin and Y. Marti, "Closed Orbit Correction of A.G. Machines using a limited Number of Magnets", CERN ISR MA/73-17 (1973).

[6] P. Collier and H. Schmickler, "The Influence of the Vertical Closed Orbit on Luminosity Performance in LEP", Proc. EPAC, London, 1994.

[7] P. Collier, "Overview of Operation in Physics During 1994", Proc. of the Fifth Workshop on LEP Performance, CERN SL/95-08 (DI).

[8] H. Burkhardt, "What Affects the Stability of LEP", Proc. of the Fifth Workshop on LEP Performance, CERN SL/95-08 (DI).

[9] I. Barnett et al., "Do we need K-Modulation in the ARCSs", Proc. of the Fifth Workshop on LEP Performance, CERN SL/95-08 (DI).

[10] P. Collier and H. Schmickler, Contribution to these procedings.

[11] G.Buur et al., "Dynamic Tune and Chromaticity Measurements in LEP", Proc. EPAC, Gif-Sur-Yvette, 1992. 